\title{
FOLIATION PRESERVING LIE GROUP ACTIONS AND CHARACTERISTIC CLASSES
}

\author{
HARUO SUZUKI ${ }^{1}$
}

\begin{abstract}
Let $\tilde{\mathscr{F}}$ be a codimension $k$ foliation of a manifold $M$ and $\mathscr{F}$ a subfoliation of $\mathscr{F}$ with codimension $q$. Let a Lie group $G$ of dimension $k$ act on $M$ transversally locally freely to $\tilde{F}$ and preserving $\mathscr{F}$. Let $\mathscr{F}^{\prime}$ be the foliation determined by $\mathscr{F}$ and the $G$-action. Then we have the following relations between exotic classes of $\mathscr{F}$ and $\mathscr{F}^{\prime}$ : $\alpha_{\mathscr{G}}\left(\left[\hat{c}_{I} c_{J}\right]\right)=\alpha_{\mathscr{F}^{\prime}}\left(\left[\hat{c}_{I} c_{J}\right]\right)$ for $I=\left(i_{1}, \ldots, i_{\lambda}\right), J=\left(j_{1}, \ldots, j_{\mu}\right), 1 \leqslant j_{\gamma}, j_{I} \leqslant q-k$, and $\alpha_{\mathscr{G}}\left(\left[\hat{c}_{I} c_{J}\right]\right)=0$ otherwise.
\end{abstract}

1. Introduction. Let $\Gamma(\xi)$ denote the set of $C^{\infty}$-sections of a vector bundle $\xi$. Let $\mathscr{F}$ be a $C^{\infty}$-foliation of a manifold $M$. We denote by $F$ the subbundle of the tangent bundle $T(M)$, which is determined by $\mathscr{F}$. It is said that a tangent vector field $Y \in \Gamma(T(M))$ preserves $\mathscr{F}$ if for each $Z \in \Gamma(F)$ we have $[Y, Z] \in \Gamma(F)$. A $k$-frame field $\left\{X_{1}, \ldots, X_{k}\right\} \subset \Gamma(T(M))$ is called transverse to $F$, if the span of $X_{1}, \ldots, X_{k}$ at each point has 0 intersection with $F$.

We say that vector fields $X_{1}, \ldots, X_{k}$ form a Lie algebra mod $\mathscr{F}$, if there exists $C^{\infty}$-functions $\alpha_{i j}^{l}$ and vector fields $Y_{i j} \in \Gamma(F), i, j, l=1, \ldots, k$, such that

$$
\left[X_{i}, X_{j}\right]=\sum_{l=1}^{k} \alpha_{i j}^{l} X_{l}+Y_{i j}
$$

Let $\xi_{i}$ be the trivial line bundle determined by $X_{i}$. If $X_{1}, \ldots, X_{k}$ form a Lie algebra $\bmod \mathscr{F}$ and each $X_{i}$ preserves $\mathscr{F}$, then the subbundle

$$
F^{\prime}=\bigoplus_{i=1}^{k} \xi_{i} \oplus F \subset T(M)
$$

is integrable and defines an extended foliation $\mathscr{F}^{\prime}$ of $M$. Let $\tilde{\mathscr{F}}$ be a $C^{\infty}$-foliation of $M$ and $\tilde{F} \subset T(M)$ the subbundle determined by $\tilde{F}$. If $F$ is a subbundle of $\tilde{F}$, then $\mathscr{F}$ is called a subfoliation of $\tilde{\mathscr{F}}$ which is denoted by $\mathscr{F} \subset \tilde{\mathscr{F}}$.

Let $\alpha_{\mathscr{F}}: H^{*}\left(W O_{q}\right) \rightarrow H^{*}(M ; \mathbf{R})$ be the map which defines the exotic classes of a foliation $\mathscr{F}$ of codimension $q[\mathrm{~B}]$. We assume all manifolds are paracompact Hausdorff $C^{\infty}$-manifolds without boundary, and all maps and bundles are of class $C^{\infty}$.

Received by the editors August 11, 1981.

1980 Mathematics Subject Classification. Primary 57R20, 57R30.

Key words and phrases. Characteristic classes, foliations, Lie group actions.

'The contents of this paper have been presented at the Annual Meeting of the Mathematical Society of Japan, Kyoto, Japan, April 6, 1981 as a preliminary report. 
THEOREM. Let $\mathscr{F}$ and $\tilde{\mathscr{F}}$ be $C^{\infty}$-foliations of a manifold $M$ such that $\mathscr{F} \subset \tilde{F}$, $\operatorname{codim} \mathscr{F}=q$ and $\operatorname{codim} \tilde{\mathcal{F}}=k$. Let $\left\{X_{1}, \ldots, X_{k}\right\}$ be a $k$-frame field transverse to $\tilde{\mathscr{F}}$. Suppose that each $X_{i}$ preserves $\mathscr{F}$ and $X_{1}, \ldots, X_{k}$ form a Lie algebra mod $\mathscr{F}$. Let $\mathscr{F}^{\prime}$ be $a$ foliation determined by $\left\{X_{1}, \ldots, X_{k}\right\}$ and the subbundle $F$ corresponding to $\mathscr{F}$. Then we have

$$
\alpha_{\mathscr{F}_{\mathcal{F}}}\left(\left[\hat{c}_{I} c_{J}\right]\right)=\alpha_{\mathscr{F}^{\prime}}\left(\left[\hat{c}_{I} c_{J}\right]\right), \quad I=\left(i_{1}, \ldots, i_{\lambda}\right), J=\left(j_{1}, \ldots, j_{\mu}\right), 1 \leqslant i_{\gamma}, j_{l} \leqslant q-k,
$$

and $\alpha_{\mathscr{G}}\left(\left[\hat{c}_{I} c_{J}\right]\right)=0$ otherwise.

This result is motivated by the theorem of Lazarov and Shulman [LS]. In §2, we prove our theorem. $\$ 3$ is devoted to show examples from lift foliations on principal bundles over foliated manifolds.

The author wishes to thank the referee for his advice.

2. Proof of the theorem. We denote the transverse (i.e., normal) vector bundles of the foliations $\mathscr{F}$ and $\mathscr{F}^{\prime}$ by $V(\mathscr{F})$ and $\left.V(\mathscr{F})^{\prime}\right)$ respectively. Since $F$ is a subbundle of $\tilde{F}$ and $M$ is paracompact Hausdorff, one can find a subbundle $\tilde{V} \subset \tilde{F}$ such that $\tilde{F}=F \oplus \tilde{V} .\left\{X_{1}, \ldots, X_{k}\right\}$ is a $k$-frame field transverse to the codimension $k$ foliation $\tilde{\mathscr{F}}$ and therefore we have

$$
T(M)=\bigoplus_{i=1}^{k} \xi_{i} \oplus F \oplus \tilde{V}
$$

By the definition of transverse vector bundles of foliations, one obtains obviously $V\left(\mathscr{F}^{\prime}\right)=T(M) / F^{\prime} \cong \tilde{V}$. Let $\tilde{\rho}: T(M) \rightarrow \tilde{V}$ denote the natural projection map. We set simply $V=V(\mathscr{F})$ and it follows that $V \cong \oplus_{i=1}^{k} \xi_{i} \oplus \tilde{V}$.

Let $\tilde{\nabla}$ be any Bott connection for $\mathscr{F}^{\prime}$ on $\tilde{V}$ and $\nabla^{\prime}$ the trivial connection with respect to the global $k$-frame field $\left\{X_{1}, \ldots, X_{k}\right\}$ on the trivial bundle $\oplus_{i=1}^{k} \xi_{i}$ : For any vector field $X \in \Gamma(T(M))$,

$$
\nabla_{X}^{\prime}\left(X_{i}\right)=0, \quad i=1, \ldots, k .
$$

We define a connection $\nabla$ on $V$ by the Whitney sum

$$
\nabla=\nabla^{\prime} \oplus \tilde{\nabla}
$$

Since $\oplus_{i=1}^{k} \xi_{i} \oplus \tilde{V}$ is a subbundle of $T(M)$, any section $s \in \Gamma(V)$ can be regarded as an element of $\Gamma\left(\oplus_{i=1}^{k} \xi_{i} \oplus \tilde{V}\right) \subset \Gamma(T(M))$. Let $\rho: T(M) \rightarrow V=T(M) / F$ be the natural projection map. For any vector field $X \in \Gamma(F)$, we have $\left[X, X_{i}\right] \in \Gamma(F)$ and hence

$$
\nabla_{X}\left(X_{i}\right)=0=\rho\left(\left[X, X_{i}\right]\right), \quad i=1, \ldots, k .
$$

Since any section $s^{\prime} \in \Gamma\left(\oplus_{i=1}^{k} \xi_{i}\right)$ is of the form $s^{\prime}=\sum_{i=1}^{k} \beta_{i} X_{i}$ where $\beta_{i}$ is a $C^{\infty}$-function on $M$ for $i=1, \ldots, k$, we obtain

$$
\nabla_{X}\left(s^{\prime}\right)=\nabla_{X}^{\prime}\left(s^{\prime}\right)=\rho\left(\left[X, s^{\prime}\right]\right) .
$$

For any section $\tilde{s} \in \Gamma(\tilde{V})$ and any vector field $X \in \Gamma(F)$, we get

$$
\nabla_{X}(\tilde{s})=\tilde{\nabla}_{X}(\tilde{s})=\tilde{\rho}([X, \tilde{s}]) .
$$


On the other hand, $\tilde{F}=F \oplus \tilde{V} \subset T(M)$ is integrable and hence the $\xi_{i}$-component of the vector field $[X, \tilde{s}]$ at each point is 0 . Therefore, for any vector field $X \in \Gamma\left(F^{\prime}\right)$, one obtains $\rho([X, \tilde{s}])=\tilde{\rho}([X, \tilde{s}])$, which means

$$
\nabla_{X}(\tilde{s})=\rho([X, \tilde{s}]) .
$$

Since any section $s \in \Gamma\left(\oplus_{i=1}^{k} \xi_{i} \oplus \tilde{V}\right)$ splits uniquely into the sum

$$
s=s^{\prime} \oplus \tilde{s}, \quad s^{\prime} \in \Gamma\left(\bigoplus_{i=1}^{k} \xi_{i}\right) \quad \text { and } \quad \tilde{s} \in \Gamma(\tilde{V}),
$$

by the formulas (1), (2) and (3), we have, for any vector field $X \in \Gamma(F)$,

$$
\begin{aligned}
\nabla_{X}(s) & =\rho\left(\left[X, s^{\prime}\right]\right)+\rho([X, \tilde{s}]) \\
& =\rho\left(\left[X, s^{\prime}+\tilde{s}\right]\right)=\rho([X, s]) .
\end{aligned}
$$

Therefore $\nabla$ is a Bott connection for $\mathscr{F}$ on $V \cong \bigoplus_{i=1}^{k} \xi_{i} \oplus \tilde{V}$.

Let $\left\{\tilde{s}_{1}, \ldots, \tilde{s}_{q-k}\right\}$ be a local $(q-k)$-frame section of $\tilde{V}$ and we set $\left\{s_{1}, \ldots, s_{q}\right\}=$ $\left\{\tilde{s}_{1}, \ldots, \tilde{s}_{q-k}, X_{1}, \ldots, X_{k}\right\}$, which is a local $q$-frame section of $V$. Let $\tilde{\theta}_{i j}, 1 \leqslant i$, $j \leqslant q-k$, be the connection forms of $\tilde{\nabla}$ with respect to $\left\{\tilde{s}_{\lambda}\right\}$. Then the connection forms $\theta_{i j}, 1 \leqslant i, j \leqslant q$, of $\nabla$ with respect to $\left\{s_{\mu}\right\}$ are given by the equations

$$
\begin{aligned}
& \theta_{i j}=\tilde{\theta}_{i j}, \quad 1 \leqslant i, j \leqslant q-k, \\
& \theta_{i j}=0, \quad i>q-k \text { or } j>q-k .
\end{aligned}
$$

In the matrix notation, we have $\theta=\left[\begin{array}{l}\tilde{\theta} \\ 0 \\ 0\end{array}\right]$, where $\tilde{\theta}=\left(\tilde{\theta}_{i j}\right), 1 \leqslant i, j \leqslant q-k$, and $\theta=\left(\theta_{i j}\right), 1 \leqslant i, j \leqslant q$.

Let $\tilde{\Omega}=\left(\tilde{\Omega}_{i j}\right)$ and $\Omega=\left(\Omega_{i j}\right)$ denote matrices of local curvature forms of $\tilde{\nabla}$ and $\nabla$ respectively. By the above equation on $\theta$ and $\tilde{\theta}$, one obtains $\Omega=\left[\begin{array}{cc}\tilde{\Omega} & 0 \\ 0 & 0\end{array}\right]$. Let $I^{j}(\mathrm{GL}(m ; \mathbf{R}))$ denote the vector space of adjoint invariant homogeneous polynomials of degree $j$ on the Lie algebra $\mathfrak{g} \mathfrak{I}(m ; \mathbf{R})$. We identify elements of $I^{j}(\mathrm{GL}(q-k ; \mathbf{R}))$ with those of $I^{j}(\mathrm{GL}(q ; \mathbf{R}))$ by the natural inclusion map $I^{j}(\mathrm{GL}(q-k ; \mathbf{R})) \subset$ $I^{j}(\mathrm{GL}(q ; \mathbf{R}))$. Let $c_{j} \in I^{j}(\mathrm{GL}(q ; \mathbf{R}))$ be Chern polynomials. (See, e.g., [B] or [C].) $c_{j}(\tilde{\Omega})$ does have meaning for $j \leqslant q-k$ and we get

$$
\begin{array}{ll}
c_{j}(\Omega)=c_{j}(\tilde{\Omega}), & 1 \leqslant j \leqslant q-k, \\
c_{j}(\Omega)=0, & j>q-k .
\end{array}
$$

We fix a Riemannian connection $\tilde{\nabla}^{0}$ on $\tilde{V}$ and then the connection $\nabla^{0}=\nabla^{\prime} \oplus \tilde{\nabla}^{0}$ on $V=\oplus_{i=1}^{k} \xi_{i} \oplus \tilde{V}$ is also a Riemannian connection. Let $\tilde{\theta}^{0}=\left(\tilde{\theta}_{i j}^{0}\right)$ be the matrix of local connection forms of $\tilde{\nabla}^{0}$ with respect to $\left\{\tilde{s}_{\lambda}\right\}$. Then the matrix $\theta^{0}$ of local connection forms of $\nabla^{0}$ with respect to $\left\{s_{\mu}\right\}$ is given by $\theta^{0}=\left[\begin{array}{cc}\tilde{\theta}^{0} & 0 \\ 0 & 0\end{array}\right]$. We form the connection

$$
\nabla^{*}=t \nabla+(1-t) \nabla^{0} \quad(t \in \mathbf{R})
$$

on the vector bundle $V \times \mathbf{R} \rightarrow M \times \mathbf{R}$. The matrix of connection forms of $\nabla^{*}$ is $\theta^{*}=t \theta+(1-t) \theta^{0}$. We denote its curvature matrix by $\Omega^{*}$. Similarly one obtains 
$\tilde{\nabla}^{*}, \tilde{\theta}^{*}$ and $\tilde{\Omega}^{*}$ on the vector bundle $\tilde{V} \times \mathbf{R} \rightarrow M \times \mathbf{R}$ and we have, for the Chern polynomials $c_{j} \in I^{j}(\mathrm{GL}(q ; \mathbf{R}))$,

$$
\begin{array}{lll}
c_{j}\left(\Omega^{*}\right)=c_{j}\left(\tilde{\Omega}^{*}\right), & & 1 \leqslant j \leqslant q-k, \\
c_{j}\left(\Omega^{*}\right)=0, & & j>q-k,
\end{array}
$$

by the same reason with (4). Then by the definition of exotic class of foliation in [B], we have easily

$$
\alpha_{\mathscr{F}}\left(\left[\hat{c}_{I} c_{j}\right]\right)=\alpha_{\mathscr{F}^{\prime}}\left(\left[\hat{c}_{I} c_{J}\right]\right) \text { for } 1 \leqslant i_{\gamma}, j_{\mu} \leqslant q-k,
$$

and $\alpha_{\mathscr{F}}\left(\left[\hat{c}_{I} c_{J}\right]\right)=0$ otherwise, which proves our theorem.

REMARK. The Roussarie foliation (see, e.g., [B]) shows that, given $\mathscr{F}$ and $X_{1}, \ldots, X_{k}$, in general, $\nabla^{\prime} \oplus \tilde{\nabla}$ is not a Bott connection and is not even $J(>q)$-homotopic to it in the sense of $[\mathbf{L}]$. The existence of $\tilde{\mathscr{F}}$ makes $\nabla^{\prime} \oplus \tilde{\nabla}$ a Bott connection.

3. Examples. Let $N$ be a manifold and $\mathscr{F}_{N}$ a $C^{\infty}$-foliation of codimension $q$ on $N$. We denote by $F_{N}$ the subbundle of $T(N)$ which is determined by $\mathscr{F}_{N}$. Let $G$ be a Lie group, $G_{0} \subset G$ a discrete subgroup and $\pi: E \rightarrow N$ a principal $G$-bundle, the structure group of which has $G_{0}$-reduction. Then there exists a homomorphism $h$ of the fundamental group $\pi_{1}(N)$ to $G_{0}$. Therefore $\pi_{1}(N)$ acts on $G$ by the left multiplication via $h$ and at the same time it acts on the universal covering manifold $\bar{N}$ of $N$ by covering transformation. It is well known that $E \cong N \times_{\pi_{1}(N)} G$. Since the diagonal action of $\pi_{1}(N)$ on $\bar{N} \times G$ preserves the foliation $\{\bar{N} \times\{g\}\}_{g \in G}$, it gives rise to a codimension $k=\operatorname{dim} G$ foliation $\tilde{\mathscr{F}}$ of $E$. Obviously $\tilde{\mathscr{F}}$ is invariant under the right action of $G$. Moreover $\mathscr{F}_{N}$ determines a foliation $\overline{\mathscr{F}}_{N}=\{\overline{\mathscr{L}}\}$ of codimension $q$ of $\bar{N}$, which is preserved by the covering transformation of $\pi_{1}(N)$. Hence the diagonal action of $\pi_{1}(N)$ on $\bar{N} \times G$ preserves the foliation $\{\overline{\mathrm{L}} \times\{g\}\}_{\mathfrak{L} \in \overline{\mathscr{F}}_{N, g} \in G}$ of codimension $q+\operatorname{dim} G$. Therefore one obtains a codimension $q+\operatorname{dim} G$ foliation $\mathscr{F}$ of $E$. $\mathscr{F}$ is also invariant under the right action of $G$. Since $F=T(\mathscr{F})$ is a subbundle of $\tilde{F}=T(\tilde{\mathscr{F}})$, we have $\mathscr{F} \subset \tilde{\mathscr{F}}$.

In our theorem, we take $E$ for $M$ and, at each point of $E$, take images of the basis vectors of the Lie algebra \&S of $G$ under the right action of $G$, for $X_{1}, \ldots, X_{k}$, $k=\operatorname{dim}\left(5\right.$. The $k$-frame field $\left\{X_{1}, \ldots, X_{k}\right\}$ is obviously transverse to $\tilde{\mathscr{F}}$ and each $X_{i}$, $i=1, \ldots, k$, preserves $\mathscr{F}$ and $\tilde{\mathscr{F}}$, because $\mathscr{F}$ and $\tilde{\mathscr{F}}$ are invariant under the right action of $G$. Moreover we have $\operatorname{codim} \tilde{\mathscr{F}}=\operatorname{dim} G=k$. Therefore it follows from our theorem that

$$
\alpha_{\mathscr{F}}\left(\left[\hat{c}_{I} c_{J}\right]\right)= \begin{cases}\alpha_{\mathscr{F}^{\prime}}\left(\left[\hat{c}_{I} c_{J}\right]\right) & \text { for } 1 \leqslant i_{\gamma}, j_{\mu} \leqslant q, \\ 0 & \text { otherwise, }\end{cases}
$$

where $\mathscr{F}^{\prime}$ is the extended foliation of $\mathscr{F}$ by $X_{1}, \ldots, X_{k}$. Since $\pi$ is a projection, $\pi$ is transverse to $\mathscr{F}_{N}$. Let $\pi^{*}\left(\mathscr{F}_{N}\right)$ denote the pullback of $\mathscr{F}_{N}$ by $\pi$ and $\pi_{*}$ denote the differential map of $\pi$. Then we have $\pi^{*}\left(\mathscr{F}_{N}\right)=\mathscr{F}^{\prime}$. For a Bott connection and a Riemannian connection in the definition of $\alpha_{\sigma^{\prime}}\left(\left[\hat{c}_{I} c_{J}\right]\right)$, one can take pullbacks by $\pi$ of those in the definition of $\boldsymbol{\alpha}_{\mathscr{F}_{N}}\left(\left[\hat{c}_{I} c_{J}\right]\right)$. Therefore we have

$$
\alpha_{\mathscr{G}^{\prime}}\left(\left[\hat{c}_{I} c_{J}\right]\right)=\pi^{*} \alpha_{\mathscr{F}_{N}}\left(\left[\hat{c}_{I} c_{J}\right]\right),
$$


and hence

$$
\alpha_{\mathscr{F}}\left(\left[\hat{c}_{I} c_{J}\right]\right)= \begin{cases}\pi^{*} \alpha_{\mathscr{F}_{N}}\left(\left[\hat{c}_{I} c_{J}\right]\right) & \text { for } 1 \leqslant i_{\gamma}, j_{\mu} \leqslant q, \\ 0 & \text { otherwise. }\end{cases}
$$

In particular, if $\alpha_{\mathscr{F}_{N}}\left(\left[\hat{c}_{I} c_{J}\right]\right)=0$ then one obtains $\alpha_{\mathscr{F}}\left(\left[\hat{c}_{I} c_{J}\right]\right)=0$. Taking the one leaf foliation of $N$ for $\mathscr{F}_{N}$, obviously one gets $\mathscr{F}=\tilde{\mathscr{F}}$ and $\alpha_{\tilde{F}}\left(\left[\hat{c}_{I} c_{J}\right]\right)=0$.

Let $f, g$ be orientation preserving diffeomorphisms of $S^{1}$ such that $f \circ g=g \circ f$. We denote the group of integers by $\mathbf{Z}$ and define an action of $\mathbf{Z} \oplus \mathbf{Z}$ without fixed points on $V=\mathbf{R}^{2} \times S^{1}$ by

$$
\begin{aligned}
& \tilde{f}\left(x_{1}, x_{2}, \theta\right)=\left(x_{1}+1, x_{2}, f(\theta)\right), \\
& \tilde{g}\left(x_{1}, x_{2}, \theta\right)=\left(x_{1}, x_{2}+1, g(\theta)\right) .
\end{aligned}
$$

The codimension 1 foliation of $V$ given by $\theta=$ const. induces a codimension 1 foliation $\mathscr{F}_{T^{3}}$ of the quotient manifold $V /(\mathrm{Z} \oplus \mathrm{Z}) \cong T$ ( 3 dimensional torus). M. R. Herman $[\mathbf{H}]$ has shown that the Godbillon-Vey class of $\mathscr{F}_{T^{3}}$ is 0 :

$$
\mathbb{S}_{v}\left(\mathscr{F}_{T^{3}}\right)=\alpha_{\mathscr{F}_{T^{3}}}\left(\left[\hat{c}_{I} c_{J}\right]\right)=0 \text {. }
$$

We set $G_{0}=\left\{\left[\begin{array}{cc} \pm 1 & 0 \\ 0 & 1\end{array}\right]\right\} \cong \mathbf{Z}_{2}$ (the group of integers $\bmod 2$ ). Then $G_{0}$ is a subgroup of $G=\mathrm{GL}(2, \mathbf{R})$. A nontrivial representation $\mathbf{Z} \oplus \mathbf{Z} \rightarrow G_{0} \cong \mathbf{Z}_{2}$ defines a left action of $\mathbf{Z} \oplus \mathbf{Z}$ on $\mathbf{G L}(2, \mathbf{R})$. Then one obtains a principal $G$-bundle

$$
V \times_{\mathbf{z} \oplus \mathbf{Z}} G=E \rightarrow T^{3}=N,
$$

the structure group of which has $G_{0} \cong \mathbf{Z}_{2}$ reduction. The 2 dimensional foliation $\mathscr{F}_{T^{3}}$ gives rise to the 2 dimensional foliation $\mathscr{F}$ of $V \times_{\mathbf{z} \oplus \mathbf{Z}} G$ stated in the beginning of this section. By our theorem, we obtain $\mathscr{S}_{v}(\mathscr{F})=0$.

\section{REFERENCES}

[B] R. Bott, Lectures on characteristic classes and foliations, Lecture Notes in Math., vol. 279, Springer-Verlag, Berlin, 1972, pp. 1-94. MR 50 \# 14777

[C] S. S. Chern, Geometry of characteristic classes, Proc. 13th Biennial Seminar of the Canadian Math. Congr. on Differential Topology, Differential Geometry and Applications, Vol. 1, Société Math. du Canada, 1972, pp. 1-49. MR 51 \#6840.

[H] M. R. Herman, The Godbillon-Vey invariant of foliations by planes of $T^{3}$, Geometry and Topology, Lecture Notes in Math., vol. 597, Springer-Verlag, Berlin and New York, 1976, pp. 294-307. MR 56 \#9548

[L] D. Lehmann, Classes caractéristiques exotiques et J-connexité des espaces de connexions, Ann. Inst. Fourier (Grenoble) 24 (1974), 267-306. MR 50 \# 14784

[LS] C. Lazarov and H. Shulman, Obstructions to foliation preserving Lie group actions, Topology 18 (1979), 255-256. MR 80m: 57020

Department of MAThematics, Hokkaido University, SAPPORo 060, JAPAN 